\title{
On the Existence of the Solution for q-Caputo Fractional Boundary Value Problem
}

\author{
Norodin A. Rangaig*, Caironesa T. Pada, Vernie C. Convicto \\ Department of Physics, Mindanao State University, 9700 Marawi City, Philippines \\ *Corresponding author: azis.norodinp6@gmail.com
}

\begin{abstract}
In this paper, we discussed the existence of a four point boundary value problem for q-fractional differential equation $D_{q}^{\alpha} x(t)=f\left(t, x(t), D_{q}^{\beta} x(t)\right)=0$ in a Banach space, particulary, using the Banach contraction principle for certain conditions on $f$.
\end{abstract}

Keywords: q-fractional integral and differential equation, q-Caputo fractional derivative, Banach contraction principle, existence of solution

Cite This Article: Norodin A. Rangaig, Caironesa T. Pada, and Vernie C. Convicto, "On the Existence of the Solution for q-Caputo Fractional Boundary Value Problem." Applied Mathematics and Physics, vol. 5, no. 3 (2017): 99-102. doi: 10.12691/amp-5-3-4.

\section{Introduction}

Fractional calculus has been always an attractive field for many researchers due to its very wide applications in the study of modeling for various fields in science and engineering, such as physics, biophysics, chemistry, controlling engineering, visco-elasticity and many more. For further details, see $[1,2,3,4,5]$ and the references therein. Years ago, the fractional equation has a been a significant progress (see [6,7] and the references therein).

The q-calculus or known as "quantum"-calculus was originally developed by Jackson [8] and some of its basic definitions and properties can be found in [9].

This study focuses on showing the existence of the solution for the following problem:

$$
\begin{gathered}
D_{q}^{\alpha} x(t)=f\left(t, x(t), D_{q}^{\beta} x(t)\right)=0, t \in[0,1], \\
x(0)=x(1)=0 \\
\epsilon_{1} x^{\prime \prime}(\Phi)=\epsilon_{2} x^{\prime \prime \prime}(\Phi)=0, \\
\epsilon_{3} x^{\prime \prime}(\Phi)+\epsilon_{4} x^{\prime \prime \prime}(\Phi)=0
\end{gathered}
$$

where $3<\alpha \leq 4, \quad \beta \leq \alpha-1$ and $D_{q}^{\alpha}$ and $D_{q}^{\beta}$ are the q-Caputo fractional Derivatives, $\epsilon_{i}(i=1,2,3,4)$ are real constants and $f$ is a continuous function on $[0,1] \times \Re^{2}$. This paper is organized as follows: in section 2 , we introduce the prelimimaries of a q-analogue and definitions of q-fractional integral and differential. In section 3, we give some hypotheses to prove the existence of solution and also using the Banach contraction priciple.

\section{Mathematical Preliminaries}

The following notations, properties, definitions, and preliminary facts will be utlized throughout this study. Let $q \in(0,1)$, then we have the q-analogue of a real number defined by [10]

$$
[\eta]_{q}=\frac{q^{\eta}-1}{q-1}
$$

The q-analogue of the power function $(\eta-\gamma)^{n}$ with $\mathrm{n}$ is a nonnegative integer is

$$
\left[(\eta-\gamma)^{n}\right]_{q}=\prod_{k=0}^{n-1}\left(\eta-q^{k} \gamma\right)
$$

in general, if $n \in \mathfrak{R}$

$$
\left[(\eta-\gamma)^{n}\right]_{q}=\eta^{n} \prod_{j=0}^{\infty} \frac{\left(\eta-q^{j} \gamma\right)}{\eta-q^{j+n} \gamma} .
$$

The q-derivative of a function $h(x)$ is defined by

$$
D_{q} h(x)=\frac{d_{q} h(x)}{d_{q} x}=\frac{f(q x)-f(x)}{x(q-1)}
$$

and the q-integral of a function $h(x)$ in the interval $[0, p]$ is given by

$$
\int_{0}^{x} h(t) d_{q} t=x(1-q) \sum_{i=0}^{\infty} h\left(x q^{i}\right) q^{i}
$$


The following an properties is useful in studying q-fractional calculus

$$
\begin{gathered}
{[u(t-s)]^{\alpha}=u^{\alpha}(t-s),} \\
{ }_{t} D_{q}(t-s)^{\alpha}=[\alpha]_{q}(t-s)^{\alpha-1} \\
\left({ }_{x} D_{q} \int_{0}^{x} h(x, t) d_{q} t\right)(x)=\int_{0}^{x}{ }_{x} D_{q} h(x, t) d_{q} t+h(q x, x) .
\end{gathered}
$$

Remark 2.1. It is to be noted that if $\alpha>0$ and $a^{\prime} \leq b^{\prime} \leq t$, then $\left(t-a^{\prime}\right)^{(\alpha)} \geq\left(t-b^{\prime}\right)^{(\alpha)}$.

\section{1. q-Fractional Integral and q-fractional Derivative}

Definition 2.2. The Reimann-Liouville q-fractional integral type for $\alpha \geq 0$ is defined by

$$
I_{q}^{\alpha} h(t)=\frac{1}{\Gamma_{q}(\alpha)} \int_{0}^{t}(t-q s)^{\alpha-1} h(s) d_{p} s
$$

where $f$ is continuous on $[0, \infty], t \in[0,1]$ and $\alpha \in \mathfrak{R}^{+}$. And the q-Gamma function is defined as

$$
\Gamma_{q}(\eta)=\int_{0}^{+\infty} x^{\eta-1} \exp (-q x) d_{p} x
$$

Definition 2.3. The Reimann-Liouville q-fractional derivative type for $\alpha \geq 0$ is defined by

$$
\left(D_{q}^{\alpha} h\right)(t)=\left(D_{q}^{[\alpha]} I_{q}^{[\alpha]-\alpha} h\right)(t)
$$

where $[\alpha]$ is the smallest possible integer greater than or equal to $\alpha$.

Definition 2.4. The Caputo q-fractional derivative type for $\alpha \geq 0$ is defined by

$$
\left(D_{q}^{\alpha} h\right)(t)=\left(I_{q}^{[\alpha]-\alpha} D_{q}^{[\alpha]} h\right)(t)
$$

where $[\alpha]$ is the smallest possible integer greater than or equal to $\alpha$.

Theorem 2.5. Let $t>0$ and $\alpha \in \mathfrak{R}^{+}$. Then the following equality holds

$$
\left(I_{q}^{[\alpha]-\alpha} D_{q}^{[\alpha]} h\right)(t)=(h)(t)-\sum_{j=0}^{[\alpha]-1} \frac{t^{j}}{\Gamma_{q}(j+1)}\left(D_{q}^{j} f\right)(0) .
$$

For further details regarding these properties, we refer the reader to [11].

\section{Mathematical Result}

Let us consider a Banach space $\chi=x: x \in C([0,1])$, $D_{q}^{\beta} \in C([0,1])$ given by the norms $\|x\|_{\chi}=\|x\|+\left\|D_{q}^{\beta} x\right\|$, $\|x\|=\sup _{t \in[0,1]}(x(t)) \quad$ and $\quad\left\|D_{q}^{\beta} x\right\|=\sup _{t \in[0,1]}\left(D_{q}^{\beta} x(t)\right)$. Then we have the following Lemmas:
Lemma 3.1. The general solution of q-fractional differential equation $D_{q}^{\alpha} x(t)=0$ for $\alpha>0$ is given by

$$
x(t)=c_{0}+c_{1} t+c_{2} t^{2}+\ldots+c_{n-1} t^{n-1},
$$

where $c_{i} \in \mathfrak{R}, n=[\alpha]+1$.

Lemma 3.2. Let $\alpha>0$. Then

$$
I_{q}^{\alpha} D_{q}^{\alpha} x(t)=x(t)+c_{0}+c_{1} t+c_{2} t^{2}+\ldots+c_{n-1} t^{n-1} .
$$

Lemma 3.3. Let $y(t) \in C([0,1])$, then the solution for the equation

$$
D_{q}^{\alpha} x(t)=y(t), t \in[0,1], 3<\alpha<4
$$

subject to the boundary conditions

$$
x(0)=x(1)=0
$$

is given by

$$
\begin{aligned}
& x(t)=\frac{1}{\Gamma_{q}(\alpha)} \int_{0}^{1}(t-q s)^{\alpha-1} f\left(s, x(s), D_{q}^{\beta} x(s)\right) d_{q} s \\
& -\frac{t}{6(b+c+a(\phi-\Phi)) \Gamma_{q}(\alpha)} \\
& \times \int_{0}^{1}(1-q s)^{\alpha-1} f\left(s, x(s), D_{q}^{\beta} x(s)\right) d_{q} s \\
& +\frac{(3 c+a(3 \phi-1)) t-3(c+a \phi) t^{2}+a t^{3}}{6(b+c+a(\phi-\Phi)) \Gamma_{q}(\alpha-2)} \\
& \times \int_{0}^{\phi}(\Phi-q s)^{\alpha-3} f\left(s, x(s), D_{q}^{\beta} x(s)\right) d_{q} s \\
& -\frac{(3 c+b(3 \phi-1)) t-3(d+b \phi) t^{2}+b t^{3}}{6(b+c+a(\phi-\Phi)) \Gamma_{q}(\alpha-3)} \\
& \quad \times \int_{0}^{\Phi}(\Phi-q s)^{\alpha-4} f\left(s, x(s), D_{q}^{\beta} x(s)\right) d_{q} s \\
& -\frac{(a(3 \phi-1)-3 b) t-3(b-a \Phi) t^{2}+a t^{3}}{6(b+c+a(\phi-\Phi)) \Gamma_{q}(\alpha-2)} \\
& \times \int_{0}^{\phi}(\phi-q s)^{\alpha-4} f\left(s, x(s), D_{q}^{\beta} x(s)\right) d_{q} s \\
& -\frac{(c(3 \phi-1)-3 d) t-3(d-c \Phi) t^{2}+c t^{3}}{6(b+c+a(\phi-\Phi)) \Gamma_{q}(\alpha-3)} \\
& \quad\left(s, x(s), D_{q}^{\beta} x(s)\right) d_{q} s
\end{aligned}
$$

where $a=\epsilon_{1} \epsilon_{2}, b=\epsilon_{2} \epsilon_{3}, c=\epsilon_{1} \epsilon_{4}, d=\epsilon_{1} \epsilon_{4}$.

Proof. By lemmas 3.1 and 3.2, general solution of (7) is given by

$$
\begin{aligned}
x(t)= & \frac{1}{\Gamma_{q}(\alpha)} \int_{0}^{t}(t-q s)^{\alpha-1} y(s) d_{q} s \\
& -c_{0}-c_{1} t-c_{2} t^{2}-c_{3} t^{3} .
\end{aligned}
$$

and using the boundary condition, we can get $c_{0}=0$

$$
c_{1}=\frac{1}{\Gamma_{q}(\alpha)} \int_{0}^{t}(1-q s)^{\alpha-1} y(s)-c_{2}-c_{3},
$$


and

$$
\begin{aligned}
& c_{2}=\frac{c+a \phi}{2(b+c+a(\phi-\Phi)) \Gamma_{q}(\alpha-2)} \int_{0}^{\Phi}(\Phi-q s)^{\alpha-3} y(s) d_{q} s \\
& -\frac{d+b \phi}{2(b+c+a(\phi-\Phi)) \Gamma_{q}(\alpha-3)} \int_{0}^{\Phi}(\Phi-q s)^{\alpha-4} y(s) d_{q} s \\
& +\frac{b-a \Phi}{2(b+c+a(\phi-\Phi)) \Gamma_{q}(\alpha-2)} \int_{0}^{\phi}(\phi-q s)^{\alpha-3} y(s) d_{q} s \\
& +\frac{d-c \phi}{2(b+c+a(\phi-\Phi)) \Gamma_{q}(\alpha-3)} \int_{0}^{\phi}(\phi-q s)^{\alpha-4} y(s) d_{q} s \\
& c_{3}=-\frac{a}{6(b+c+a(\phi-\Phi)) \Gamma_{q}(\alpha-2)} \int_{0}^{\Phi}(\Phi-q s)^{\alpha-3} y(s) d_{q} s \\
& +\frac{b}{6(b+c+a(\phi-\Phi)) \Gamma_{q}(\alpha-3)} \int_{0}^{\Phi}(\Phi-q s)^{\alpha-4} y(s) d_{q} s \\
& +\frac{a}{6(b+c+a(\phi-\Phi)) \Gamma_{q}(\alpha-2)} \int_{0}^{\phi}(\phi-q s)^{\alpha-3} y(s) d_{q} s \\
& +\frac{c}{6(b+c+a(\phi-\Phi)) \Gamma_{q}(\alpha-3)} \int_{0}^{\phi}(\phi-q s)^{\alpha-4} y(s) d_{q} s .
\end{aligned}
$$

Substituting these obtained constant to equation (9) and making use of the lemma (3.3), we can have (8).

\subsection{Existence of the Solution}

In this section, we will show that there exist a solution of the problem (1) and show the contribution of the term $D_{q}^{q} x(t)$ on the solution. Suppose the following quantities:

$$
\begin{aligned}
& N_{1}=\frac{|b+c+a(\phi-\Phi)|+1}{6|b+c(\phi-\Phi)| \Gamma_{q}(\alpha+1)} \\
& +\frac{\left[\begin{array}{l}
(|3 c+a(3 \phi-1)|+3|c+a \phi|+|a|) \Phi^{\alpha-2} \\
+(|a(3 \Phi-1)-3 b|+3|b-a \Phi|+|a|) \phi^{\alpha-2}
\end{array}\right]}{6|b+c+a(\phi-\Phi)| \Gamma_{q}(\alpha+1)} \\
& +\frac{\left[\begin{array}{l}
(|3 d+b(3 \phi-1)|+3|d+b \phi|+|b|) \Phi^{\alpha-3} \\
+(|c(3 \Phi-1)-3 d|+3|d-c \Phi|+|c|) \phi^{\alpha-3}
\end{array}\right]}{6|b+c+a(\phi-\Phi)| \Gamma_{q}(\alpha-2)} \\
& N_{2}=\frac{1}{\Gamma_{q}(\alpha-\beta+1)}+\frac{1}{6|b+c(\phi-\Phi)| \Gamma_{q}(\alpha+1) \Gamma_{q}(2-\beta)} \\
& +\frac{|3 c+a(3 \phi-1)| \Phi^{\alpha-2}+|a(3 \Phi-1)-3 b| \phi^{\alpha-2}}{6|b+c+a(\phi-\Phi)| \Gamma_{q}(\alpha-1) \Gamma_{q}(2-\beta)} \\
& +\frac{|c+a \phi| \Phi^{\alpha-2}+|b-a \Phi| \phi^{\alpha-2}}{|b+c+a(\phi-\Phi)| \Gamma_{q}(\alpha-1) \Gamma_{q}(3-\beta)} \\
& +\frac{|3 d+b(3 \phi-1)| \Phi^{\alpha-3}+|c(3 \Phi-1)-3 d| \phi^{\alpha-3}}{6|b+c+a(\phi-\Phi)| \Gamma_{q}(\alpha-2) \Gamma_{q}(2-\beta)} \\
& +\frac{|a|\left(\Phi^{\alpha-2}+\phi^{\alpha-2}\right)}{|b+c+a(\phi-\Phi)| \Gamma_{q}(\alpha-1) \Gamma_{q}(4-\beta)}
\end{aligned}
$$

$$
\begin{aligned}
& +\frac{|d+b \phi| \Phi^{\alpha-3}+|d-c \Phi| \phi^{\alpha-3}}{6|b+c+a(\phi-\Phi)| \Gamma_{q}(\alpha-2) \Gamma_{q}(2-\beta)} \\
& +\frac{|b| \Phi^{\alpha-2}+|c| \phi^{\alpha-2}}{|b+c+a(\phi-\Phi)| \Gamma_{q}(\alpha-2) \Gamma_{q}(4-\beta)} .
\end{aligned}
$$

Following the hypotheses of Houas and Dahmani [3], we can consider the following the hypothesis:

Hypothesis 3.4. The function $f: C([0,1]) \times \mathfrak{R}^{2} \rightarrow \mathfrak{R}$ is continous.

In accordance also to the work of El-Shahed and Al-Yami [12], we have

Hypothesis 3.5. Assume that there exist a nonegative constant $M>0$ such that

$$
|f(t, x, y)| \leq M ; t \in[0,1], x, y \in \mathfrak{R} .
$$

Hypothesis 3.6. There exist a nonnegative function $u, v$ on $[0,1]$, such that for $t \in[0,1],(x, y),\left(x_{1}, y_{1}\right) \in \mathfrak{R}^{2}$

$$
\left|f(x, y)-f\left(x_{1}, y_{1}\right)\right| \leq u(t)\left|x-x_{1}\right|+v(t)\left|y-y_{1}\right|
$$

where $\Omega=\sup _{t \in[0,1]}|u(t)|$ and $\tilde{\Omega}=\sup _{t \in[0,1]}|v(t)|$.

Theorem 3.7. Suppose hypothesis (3.6) holds if

$$
\left(N_{1}+N_{2}\right)(\Omega+\tilde{\Omega})<1
$$

then the solution for the problem (1) exist on $[0,1]$. Proof. Consider a q-operator

$$
F_{q}: C([0,1], \Re) \rightarrow C([0,1], \Re)
$$

such that, it is defined as

$$
\begin{aligned}
& F_{q} x(t)=\frac{1}{\Gamma_{q}(\alpha)} \int_{0}^{t}(t-q s)^{\alpha-1} f\left(s, x(s), D_{q}^{\beta} x(s)\right) d_{q} s \\
& -\frac{t}{6(b+c+a(\phi-\Phi)) \Gamma_{q}(\alpha)} \\
& \times \int_{0}^{1}(1-q s)^{\alpha-1} f\left(s, x(s), D_{q}^{\beta} x(s)\right) d_{q} s \\
& +\frac{(3 c+a(3 \phi-1)) t-3(c+a \phi) t^{2}+a t^{3}}{6(b+c+a(\phi-\Phi)) \Gamma_{q}(\alpha-2)} \\
& \times \int_{0}^{\phi}(\Phi-q s)^{\alpha-3} f\left(s, x(s), D_{q}^{\beta} x(s)\right) d_{q} s \\
& -\frac{(3 d+b(3 \phi-1)) t-3(d+b \phi) t^{2}+b t^{3}}{6(b+c+a(\phi-\Phi)) \Gamma_{q}(\alpha-3)} \\
& \times \int_{0}^{\Phi}(\Phi-q s)^{\alpha-4} f\left(s, x(s), D_{q}^{\beta} x(s)\right) d_{q} s \\
& -\frac{(a(3 \Phi-1)-3 b) t-3(b \Phi-a) t^{2}+a t^{3}}{6(b+c+a(\phi-\Phi)) \Gamma_{q}(\alpha-2)} \\
& \times \int_{0}^{\phi}(\phi-q s)^{\alpha-3} f\left(s, x(s), D_{q}^{\beta} x(s)\right) d_{q} s \\
& -\frac{(c(3 \Phi-1)-3 d) t-3(d-c \Phi) t^{2}+c t^{3}}{6(b+c+a(\phi-\Phi)) \Gamma_{q}(\alpha-3)} \\
& \times \int_{0}^{\phi}(\phi-q s)^{\alpha-4} f\left(s, x(s), D_{q}^{\beta} x(s)\right) d_{q} s .
\end{aligned}
$$


In which we must show that $F_{q}$ is a contraction

For any $x, y \in C([0.1], \Re)$, using Hypothesis 3.6 we can obtain

$$
\begin{aligned}
& \left|F_{q} x(t)-F_{q} y(t)\right| \\
& \leq \frac{1}{\Gamma_{q}(\alpha)} \int_{0}^{t}(t-q s)^{\alpha-1}\left|\begin{array}{l}
f\left(s, x(s), D_{q}^{\beta} x(s)\right) \\
-f\left(s, y(s), D_{q}^{\beta} y(s)\right.
\end{array}\right| d_{p^{s}} \\
& -\frac{t}{6|b+c+a(\phi-\Phi)| \Gamma_{q}(\alpha)} \\
& \times \int_{0}^{1}(1-q s)^{\alpha-1}\left|\begin{array}{l}
f\left(s, x(s), D_{q}^{\beta} x(s)\right) \\
-f\left(s, y(s), D_{q}^{\beta} y(s)\right)
\end{array}\right| d_{q} s \\
& +\frac{|3 c+a(3 \phi-1)| t-3|c+a \phi| t^{2}+|a| t^{3}}{6|b+c+a(\phi-\Phi)| \Gamma_{q}(\alpha-2)} \\
& \times \int_{0}^{\phi}(\phi-q s)^{\alpha-3}\left|\begin{array}{l}
f\left(s, x(s), D_{q}^{\beta} x(s)\right) \\
-f\left(s, y(s), D_{q}^{\beta} y(s)\right)
\end{array}\right| d_{q} s \\
& -\frac{|3 d+b(3 \phi-1)| t-3|d+b \phi| t^{2}+|b| t^{3}}{6|b+c+a(\phi-\Phi)| \Gamma_{q}(\alpha-3)} \\
& \times \int_{0}^{\Phi}(\Phi-q s)^{\alpha-4}\left|\begin{array}{l}
f\left(s, x(s), D_{q}^{\beta} x(s)\right) \\
-f\left(s, y(s), D_{q}^{\beta} y(s)\right.
\end{array}\right| d_{q} s \\
& -\frac{|a(3 \Phi-1)-3 b| t-3|b-a \Phi| t^{2}+|a| t^{3}}{6|b+c+a(\phi-\Phi)| \Gamma_{q}(\alpha-2)} \\
& \times \int_{0}^{\phi}(\phi-q s)^{\alpha-3}\left|\begin{array}{l}
f\left(s, x(s), D_{q}^{\beta} x(s)\right) \\
-f\left(s, y(s), D_{q}^{\beta} y(s)\right)
\end{array}\right| d_{q} s \\
& -\frac{|c(3 \Phi-1)-3 d| t-3|d-c \Phi| t^{2}+|c| t^{3}}{6|b+c+a(\phi-\Phi)| \Gamma_{q}(\alpha-3)} \\
& \times \int_{0}^{\phi}(\phi-q s)^{\alpha-4}\left|\begin{array}{l}
f\left(s, x(s), D_{q}^{\beta} x(s)\right) \\
-f\left(s, y(s), D_{q}^{\beta} y(s)\right.
\end{array}\right| d d_{q} s .
\end{aligned}
$$

To reduce the expression, we use Hypothesis (3.5) and (3.6) and simplifying further we can obtain the relation

$$
\begin{aligned}
& \left|F_{q} x(t)-F_{q} y(t)\right| \\
& \leq N_{1}(\Omega+\tilde{\Omega})\left(\|x-y\|+\left\|D_{q}^{\beta} x-D_{q}^{\beta} y\right\|\right) .
\end{aligned}
$$

Furthermore, to finally show the contraction of $F_{q}$. We can use the definition of a q-Caputo Fractional Derivative
$[3,11]$, by lemma (3.3) and lastly the hypotheses (3.4-3.6). we can finally obtain

$$
\begin{aligned}
& \left\|F_{q} x(t)-F_{q} y(t)\right\|_{C([0.1], \Re)} \\
& \leq\left(N_{1}+N_{2}\right)(\Omega+\tilde{\Omega})\left(\|x-y\|+\left\|D_{q}^{\beta} x-D_{q}^{\beta} y\right\|\right) .
\end{aligned}
$$

\section{Conclusion}

Hence, by the hypothesis (3.6), we deduced that $F_{q}$ is a consequence of Banach contraction principle and the solution of equation (1) exist for $t \in[0,1]$. For a Generic case, we can say that the contraction obtained in [12], which is a three point q-fractional boundary problem, coincides with the obtained result in this study.

\section{Acknowledgements}

The authors would like to thank the Department of Physics, College of Natural Sciences and Mathematics, Minadanao State University-Main Campus, Marawi City.

\section{References}

[1] Z. Bai, Y Zhang. Solvability of Fractional Three-Point Boundary Value Problems with Nonlinear growth, Appl. Math. Comp., 218(5), pp. 1719-1725, 2011.

[2] M.E Bengrine, Z. Dahmani, Boundary Value Problems for Fractional Differential Equations, Int. J. Open Prob. Comp. Math. 5(4), 2012.

[3] M. Houas, Z. Dahmani, New Results for Caputo Boundary Value Problem, Am. J. Comp. App. Math, 3(3), pp. 143-161, 2013.

[4] M. Houas, Z. Dahmani, New Results for Differential Equations of Arbitrary Order, IJMMS Journal Int'l Press.

[5] A.A. Kilbas, S.A. Marzan, Nonlinear Differential Equation with the Caputo Fractional Derivative in the Space of Continuously differentiable function, Diff. Eq., 41(1), pp. 84-89, 2005.

[6] A.M El-Sayed, Nonlinear Functional Differential Equations of Arbitrary Orders, Nonlinear Analysis, 33(2), pp. 181-186, 1998.

[7] S. Zhang, S. Chen, J. Lu, Upper and Lower Solution Method for fourth-order Four-point Boundary Value Problems, J. Diff. Eq., 2(36), pp. 12-19, 2006.

[8] F. H. Jackson, On q-Functions and Certain Difference Operator, Trans. Roy. Soc., Edinburgh 46, pp. 253-281, 1908.

[9] F. H. Jackson, On q-Definite Integrals, Quart. J. Pure Appl. Math, 41, pp.193-203, 1910.

[10] V. Kac, P. Cheung, Quantum Calculus, Springer-Verlag, New York, 2002

[11] M. S. Stankovic, et. al, On q-fractional derivatives of ReimannLiouville and Caputo Type, arXiv:0909, 2009.

[12] M. El-Shahed, M. Al-Yami, On the Existence and Uniqueness of Solution for Q-fractional Boundary Value Problem, Int'l J. Math. Anal., vol 5. no. 33, pp. 1619-1630, 2011. 